\title{
Educación filosófica para la igualdad de género y la sostenibilidad medioambiental: el pensamiento de Ann Sharp*
}

\author{
Philosophical Education for Gender Equality and \\ Environmental Sustainability: Ann Sharp's Thinking
}

GEORGINA AIMÉ TAPIA GONZÁLEZ**

\begin{abstract}
Resumen: Los reiterados cuestionamientos sobre la pertinencia de mantener cursos de Filosofía en la educación secundaria y media superior, se ciernen como una amenaza sobre las sociedades democráticas. Reducir o eliminar las materias filosóficas del currículum implica dejar de lado la potencialidad crítica del pensamiento en un contexto de crisis generalizada: ecológica, económica, social y, sobre todo, de sentido. El presente artículo representa un acercamiento a la propuesta educativa de la filósofa que, junto a Matthew Lipman, contribuyó a conformar uno de los proyectos pedagógicos que ha tenido mayor impacto en el ámbito educativo, me refiero a Ann Sharp. Mi objetivo es mostrar la relevancia del legado de esta pensadora, que vislumbró la posibilidad de una educación filosófica para la igualdad de género y la sostenibilidad medioambiental, tan necesaria hoy.
\end{abstract}

Palabras clave: Filosofía, educación, género, ética ecológica.

\begin{abstract}
The ongoing questionings in relation the pertinence of maintaining philosophical courses in high and middle school loom dangerously over democratic societies. To reduce or eliminate philosophical classes form the syllabus implies leaving aside the critical potentiality of thinking in a context of generalized crisis: ecological, economic, social, and above all, of sense. This article represents an approach to the Ann Sharp's educational proposal that, in collaboration with Matthew Lipman, has contributed to shape one of the teaching projects that has had one the greatest effects in the educational arena. My aim is to show the relevance of this thinker's legacy, who envisioned the possibility of a philosophical education that fosters gender equality and environmental sustainability, so important nowadays.
\end{abstract}

Keywords: Philosophy, Education, Gender, Ecological Ethics.

Recibido: 04/11/2015. Aceptado: 09/12/2015.

* Este artículo forma parte del proyecto de investigación: "Filosofía para/con niñas/os y jóvenes (FpN y J): aproximaciones teóricas y propuestas prácticas desde una mirada ecofeminista”, financiado por el Programa para el Desarrollo Profesional Docente (PRODEP), para el tipo superior, Secretaría de Educación Pública, México.

** Universidad de Colima, México. Profesora en la Facultad de Pedagogía y en la Escuela de Filosofía. Principales líneas de investigación: Filosofía feminista y estudios de género, ecofeminismo y ética ecológica. Publicaciones recientes: (2015) “Aportaciones de las mujeres indígenas al diálogo entre filosofía y ecología” en Puleo, Alicia (ed.), Ecología y género en diálogo interdisciplinar, Madrid, Plaza y Valdés, pp. 263-277. (2016) "La filosofía feminista de Edith Stein”, en González Di Pierro, E. (ed). Edith Stein: una filósofa del siglo XX para el siglo XXI, Biblos, Buenos Aires (en prensa).Correo electrónico: georgina_tapia@ucol.mx 


\section{Introducción}

El proyecto de Filosofía para Niñas/os y Jóvenes (FpN y J) representa una de las tendencias más relevantes dentro de la filosofía de la educación. A partir del currículum desarrollado por M. Lipman, pensadoras/es pertenecientes a geografías diversas han realizado propuestas originales que responden a problemáticas surgidas de sus propios contextos: cosmovisiones de los pueblos originarios, multiculturalidad, crisis socioambiental, entre otras. Al respecto, es importante destacar que desde el origen de $\mathrm{F}$ pN y J estuvo presente una filósofa que aportó su visión feminista a las propuestas de M. Lipman, me refiero a A. Sharp. Esta autora ha contribuido a la visibilización de temas tradicionalmente desestimados en el pensamiento occidental. De acuerdo con ella, la vocación dialógica de la filosofía debe incluir las voces de las niñas, los niños, las mujeres y de muchos «otros» a quienes no se les ha reconocido como sujetos del filosofar.

El objetivo de este ensayo es recuperar el legado de A. Sharp para una educación filosófica capaz de integrar a «esos otros». Esta filósofa llegó incluso a preguntarse si era posible considerar a los seres no humanos como interlocutores en las comunidades de indagación sobre temas relacionados con la ética ecológica. Todo esto hace posible sostener que sus ideas aportan elementos relevantes para la comprensión de algunos de los problemas más acuciantes de nuestro tiempo, como el sexismo, el maltrato infantil y la crisis ecológica. Ahora bien, lo anterior se desarrollará a través de los siguientes apartados: en el primero, se abordan los rasgos principales de $\mathrm{FpN}$ y J; en el segundo, se reflexiona sobre los vínculos existentes entre FpN y J y filosofía feminista; finalmente, en el tercero, se plantean algunas alternativas para una educación ambiental comprometida con la igualdad de género.

\section{Pensamiento crítico y educación: un acercamiento a la Filosofía para Niñas/os y J $(\mathbf{F p N}$ y J)}

Ann Sharp y Mathew Lipman han sido los creadores de una innovadora propuesta dentro de la filosofía de la educación. Ambos llegaron a la conclusión de que una enseñanza auténticamente filosófica debía ser el fundamento del currículum de todos los niveles educativos. De acuerdo con ellos, la ausencia del pensamiento crítico dentro de las distintas materias, incluso dentro de la propia filosofía, ha dado como resultado estudiantes que ingresan a la universidad sin haber desarrollado habilidades básicas en el ámbito de la lógica, así como de la expresión oral y escrita; jóvenes que se convierten en adultos incapaces de dar razón de los criterios que rigen su conducta en la vida diaria. Por ello, dedicaron parte de su vida al desarrollo de un proyecto educativo dirigido a niños/as y adolescentes, que se ha extendido por diferentes partes del mundo, convirtiéndose en una de las corrientes pedagógicas más prometedoras para el siglo XXI.

Si bien una educación filosófica resulta insuficiente para solucionar la «crisis de crisis» que caracteriza a nuestro mundo -social, ecológica, política, económica y, sobre todo, de sentido-, es innegable su capacidad para impulsar alternativas que contribuyan a ello. La filosofía para/con niños/as y jóvenes cuestiona algunos de los prejuicios más arraigados 
en la historia de la filosofía y de la educación, al mismo tiempo que aporta elementos para la conformación de sociedades plurales, democráticas, con paridad de género y ecológicamente responsables.

En primer lugar, la FpN y J muestra los límites de una concepción bastante extendida sobre la filosofía, como saber obtuso e inaccesible para la mayor parte de las personas. Frente a esta idea, sostiene que la «venerable madre de todas las ciencias» debe estar al alcance de todos los seres humanos, contribuyendo a convertir los centros educativos en comunidades donde se estimule el pensamiento crítico, creativo y moral. A juicio de Tomás Miranda, FpN y J es «un proyecto educativo que tiene como objetivo enseñar a pensar, a sentir y a vivir de un modo riguroso, crítico, creativo, solidario y cuidadoso» $(2007,1)$. Cada ser humano necesita desarrollar sus capacidades de razonamiento, formación de conceptos, juicios morales y creatividad, para el desenvolvimiento pleno de su humanidad. Desde esta perspectiva, la filosofía renuncia a cualquier pretensión elitista, poniéndose al servicio de la sociedad.

M. Lipman considera que el ideal de la educación filosófica debe guiarse en torno al cultivo del pensamiento crítico y el ejercicio de la racionalidad dentro de una comunidad de diálogo. En sus palabras: «el pensamiento crítico es un pensamiento que 1) facilita el juicio porque 2) se basa en criterios 3) es autocorrectivo y 4) sensible al contexto» (1998, 174). La sabiduría, entendida como una forma de pensamiento razonable y reflexivo, está en la base de los buenos juicios. Un juicio es bueno si se fundamenta en criterios sólidos, es decir, si aporta razones valiosas para sostener su objetividad.

Uno de los objetivos más importantes que se persigue al convertir el salón de clases en una comunidad de indagación, además del mejoramiento del clima moral, es el interés por descubrir las debilidades y los errores de los propios juicios para poder rectificarlos. El diálogo con otros/as posibilita el reconocimiento de la propia falibilidad y presenta alternativas para la autocorrección. La sensibilidad al contexto implica la apertura ante excepciones, irregularidades, contingencias, traducciones difíciles, etcétera, esto es, el reconocimiento de que cada circunstancia conlleva una densidad de significado que no es fácilmente transferible, de ahí que lo que se asume como «verdadero» dentro de una situación determinada, podría no serlo en otra (Lipman, 1998; Miranda, 2007).

Para M. Lipman, el pensamiento crítico requiere del cultivo de la racionalidad, pero también de la razonabilidad. La primera apunta al proceso cognitivo que permite disponer de los medios más adecuados para lograr los fines propuestos; en tanto que la segunda se refiere a la lógica de las buenas razones aplicada a la vida y los actos de los seres humanos. Al respecto, Tomás Miranda argumenta que una de las mayores contribuciones que puede hacer la filosofía a la educación consiste precisamente en la formación de personas razonables: «Si la racionalidad puede ser considerada la idea regulativa de la investigación científica, la razonabilidad lo es del proceso educativo» (2007, 7). Aquí podemos preguntarnos ¿Hacia dónde nos conduce una racionalidad que renuncia a la razonabilidad? ¿Hacia una educación sin filosofía?

Sin una dimensión que incluya la indagación filosófica, no sólo la educación pierde sentido, sino también la vida política queda vacía de todo contenido significativo. Formar seres humanos que han aprendido el hábito de razonar, escuchar, dialogar, justificar sus juicios morales y ser flexibles ante distintos contextos constituye una de las mayores aportaciones de la filosofía a la conformación de comunidades democráticas. Eliminar la filosofía de la 
escuela, o convertirla en un cúmulo de información desconectada de las necesidades más apremiantes del mundo actual, equivale a renunciar a lo que se ha considerado el distintivo del animal humano, a saber, su capacidad de pensar sobre el pensamiento y la vida propios.

En segundo lugar, la FpN y J abre nuevos horizontes sobre las potencialidades lógicas, morales y creativas de la infancia. Un lugar común dentro de la historia de las ideas ha sido la creencia de que los/as niños/as son incapaces de reflexión y pensamiento crítico. Por una parte, se les concibe como «personitas» relegadas al mundo de la fantasía, el ensueño, la irrealidad; no es casualidad que la educación que se les imparte, tanto de manera formal como informal, desestime su plena humanidad. Por otra, millones de ellos/as enfrentan situaciones de pobreza, marginación, violencia y abuso sexual. ¿Por qué negarles la filosofía si ésta puede contribuir a educarlos/as en un sentido profundo? ¿Acaso sus preguntas, sus experiencias, sus visiones del mundo están desprovistas de relevancia filosófica? ¿Qué dicen las voces de los/as niños/as y jóvenes en medio del caos en que vivimos actualmente? A. Sharp y M. Lipman justifican su filosofía dirigida a los/as niños/as a través de los siguientes argumentos:

La filosofía comienza en el asombro, y los niños en sus primeros años son siempre curiosos y se preguntan por el significado de los conceptos; se preguntan cómo funcionan las cosas, por qué estamos aquí en este mundo, de dónde venimos, hacia dónde vamos, etc. La filosofía apunta hacia la comprensión y también los niños desean comprender: comprenderse a sí mismos, comprender el mundo en el que viven; y, sobre todo, intentan encontrar sentido en medio de lo que W. James llamó «esta confusión de moda y en auge» (A. Sharp y M. Lipman en Pineda, 2006, 1).

Los estudios realizados por Piaget y Vygotski en torno al desarrollo cognitivo constituyen dos de los fundamentos teóricos más relevantes de la FpN y J. Si bien Piaget sostenía que antes de los 12 años la mayor parte de los seres humanos aún no puede pensar filosóficamente, sus investigaciones contribuyeron a poner sobre la mesa de discusión la capacidad de los/as infantes para reflexionar en torno a su propio pensamiento. Por su parte, Vygotski llegó a hipótesis muy diferentes a las de su contemporáneo. Según los estudios realizados por este autor, la infancia se caracteriza por tener niveles de desarrollo flexibles que pueden ser estimulados por la acción de los/as docentes y los/as compañeros/as, es decir, un/a menor que recibe los incentivos adecuados en la escuela, alcanza el siguiente nivel de desarrollo aunque todavía no tenga la edad necesaria (Waksman y Kohan, 2000).

Después de haber estudiado a los grandes teóricos de la niñez, y bajo la influencia de las ideas de Dewey sobre la comunidad de investigación (Waksman y Kohan, 2000), así como a través de su experiencia directa en el trato con niños/as y jóvenes, los creadores del proyecto de FpN y J llegaron a la conclusión de que estos/as no sólo son aptos/as para pensar filosóficamente si se les dan las herramientas adecuadas para hacerlo, sino que también pueden aportar ideas interesantes para la reflexión. M. Lipman escribió su primer novela filosófica, El descubrimiento de Harry $(1987)^{1}$, para adolescentes de 12 años, pero A. Sharp le dio razones suficien-

1 En castellano se consultaron las siguientes versiones: Lipman, Matthew (2006), Harry Prime, Traducción de Maximiliano Papandrea, Buenos Aires, Manantial. Lipman, Matthew (2014), El descubrimiento de Filio episteme, traducción y adaptación para México de Eugenio Echeverría, Chiapas, México, Centro Latinoamericano de Filosofía para Niños. 
tes de que, incluso, a edades muy tempranas era posible incentivar el pensamiento filosófico, porque los seres humanos se caracterizan por intentar comprender y encontrar un para qué del mundo que les rodea. Lo anterior fue demostrado por A. Sharp y L. Splitter en su obra Hospital de muñecas (1996), diseñada para hacer filosofía en el jardín de infancia. Los resultados fueron sorprendentes: niños/as muy pequeños/as comenzaron a formularse preguntas sobre el ser de las cosas, el sentido de la vida, el misterio de la muerte, etcétera, y se aventuraron a pensar estas cuestiones en la comunidad conformada por sus pares y su profesor/a. Estas prácticas pedagógicas hicieron patente que: «A través de los niños, la filosofía puede reaprender la importancia de las cuestiones metafísicas por medio de las cuales tratamos de comprendernos a nosotros mismos y comprender el mundo (...). Además, y puesto que su proceso de socialización apenas está comenzando, los puntos de vista filosóficos de los niños tienden a ser frescos, originales y llenos de posibilidades» (A. Sharp y M. Lipman en Pineda, 2006, 1).

En tercer lugar, la FpN se propone revolucionar la educación. Siguiendo a Dewey, Lipman considera que la ineficacia de los programas educativos se debe principalmente a que se ha dado prioridad a enseñar los resultados alcanzados por las ciencias, dejando de lado los procesos. Si queremos personas pensantes y responsables, es imprescindible potenciar las capacidades cognitivas, creativas y morales de los/as niños/as, de ahí que la escuela no pueda seguir siendo solamente el espacio en el que se transmiten conocimientos, sino que tiene que convertirse en un lugar en el que se estimule el desarrollo del pensamiento complejo. Nuestro autor se propuso recuperar la concepción socrática de la filosofía, entendida como la capacidad humana para dialogar sobre asuntos de interés común que se relacionan con nuestras experiencias y contextos, para ponerla al alcance los los/as niños/as y los/as jóvenes (Nussbaum, 2010)². Para lograrlo, pensó que lo más adecuado era introducir los temas filosóficos utilizando historias noveladas que presenten personajes y problemáticas similares a las experimentadas por aquéllos/as a quienes iban dirigidas.

La propuesta de M. Lipman y A. Sharp (2002) significa un gran cambio en los libros de texto, en las formas tradicionales de enseñar y en los roles del/a maestro/a. El centro del proceso de aprendizaje es la comunidad de indagación integrada por pares y guiada por un/a profesional sólidamente preparado/a en el ámbito de la filosofía. Siguiendo los pasos de Sócrates, el/la profesor/a estimula la búsqueda conjunta de respuestas ante las problemáticas planteadas, llevando a sus estudiantes a examinar sus propios juicios, con el propósito de que descubran los supuestos que subyacen a ellos y se aventuren a formular ejemplos e intenten aportar evidencias. Como apunta Diana Hoyos: «para Lipman la filosofía es la disciplina llamada a darle mayor sentido al currículo educativo» $(2010,161)$. El programa se propone desarrollar en los/as niños/as y jóvenes las habilidades fundamentales de la lógica, además de estimular el pensamiento creativo y el moral. Para «pensar bien» es necesario examinar los criterios de los que se ha partido, analizar argumentos, develar errores e inconsistencias, pero también se requiere de imaginación, expresividad, originalidad y de la capacidad de cuidar de sí mismo/a y de los/as demás, de valorar lo valioso y actuar en consecuencia. Es evidente que todas estas habilidades se requieren en cada una de las asignaturas y no sólo en las de filosofía (Hoyos, 2010).

2 Sobre pedagogía socrática véase Nussbaum, Martha. (2010), Sin fines de lucro. Por qué la democracia necesita de las humanidades. Trad. de María Victoria Rodil, Buenos Aires/Madrid, Katz. 


\section{Importancia de visibilizar a las niñas y las adolescentes dentro del programa de FpN y J}

El proyecto de $\mathrm{FpN}$ y $\mathrm{J}$ ha sido muy bien recibido alrededor del mundo y los libros de $\mathrm{M}$. Lipman se han traducido a diferentes idiomas ${ }^{3}$. En el caso específico del castellano -al igual que en otras lenguas que pluralizan utilizando el género masculino-, las niñas parecen haber sido borradas de la propuesta. Si bien es cierto que algunos de los trabajos sobre FpN y J utilizan un lenguaje incluyente, y que, como veremos más adelante, A. Sharp le concedió una importancia capital al tema del feminismo, las problemáticas específicas sobre los vínculos entre género e infancia no han ocupado un lugar prioritario en el programa.

Las razones para tomar este problema con seriedad han sido expuestas por la UNICEF (2014) a través de los siguientes datos: «Alrededor de 120 millones de niñas menores de 20 años en el mundo (casi una de cada 10) han sufrido agresiones sexuales» (Las estadísticas clave incluyen, párr. 2). Y más adelante continúa: «la información disponible señala que en algunos países, casi 7 de cada 10 niñas entre los 15 y los 19 años de edad que han sido víctimas de la violencia física y/o del abuso sexual, nunca buscaron ayuda: muchas de ellas mencionaron que no lo percibieron como un abuso o un problema» (párr. 3). Ante la gravedad de la situación, el Secretario General de las Naciones Unidas, Ban Ki-Moon, dedicó el Día Internacional de la Niña 2014 al tema: «Empoderar a las adolescentes: poner fin al ciclo de la violencia». Los caminos que propone para lograrlo incluyen potenciar capacidades y alternativas de vida que permitan a las niñas y jóvenes desarrollarse como seres humanos en igualdad de circunstancias que los varones. Para ello es imprescindible visibilizar las diversas formas de violencia de que son objeto, garantizar su libre acceso a la salud, la educación, la tecnología, y a la vida cívica, económica y política (Naciones Unidas, 11 de octubre de 2014).

Ahora bien, ¿qué puede aportar FpN y J ante la compleja problemática que plantea la desigualdad de género?

El vínculo entre FpN y feminismo fue señalado por A. Sharp desde los inicios de la propuesta educativa que contribuyó a construir junto a M. Lipman. Nuestra pensadora considera que existen varios puntos de confluencia entre estas dos perspectivas filosóficas: por una parte, coinciden en su interés hacia las voces tradicionalmente silenciadas, subrayan la importancia de la experiencia personal y la narrativa como fuentes de conocimiento válidas, cuestionan los alcances de la «objetividad» y la «universalidad» y asumen un compromiso ético con la pluralidad y la inclusión; por otra, proponen una pedagogía fundada en el cuidado de las/os otras/os, el diálogo y la búsqueda de sentido, valoran el trabajo colaborativo y conciben a la Filosofía como una indagación continua, abierta a las/os niñas/os, las mujeres y las personas pertenecientes a diversas etnias y culturas. En palabras de la autora, ambas «subrayan la necesidad de ofrecer un sistema educativo en el cual los valores y las actitudes que subyacen al sexismo puedan ser puestos en cuestión y replanteados» (Sharp, 1989, 1997; Splitter y Sharp, 1996, 275).

3 María Teresa de la Garza argumenta que gracias a A. Sharp, el programa de FpN y J se extendió por todo el mundo. Fue ella la que visitó diferentes países y presentó esta propuesta a filósofas/os y pedagogas/os de todos los continentes: «si pensamos a FpN como una vela, Lipman representa la flama azul, que mantiene encendida la llama, y Sharp la flama naranja, que es la que la extiende». De la Garza, María Teresa (23 de junio de 2015), Entrevista realizada por Tapia González, Georgina Aimé, publicada en (2017). Pensar Juntos. Revista Iberoamericana de Filosofía para Niños, 1, 83-97. 
Llama la atención que tanto la filosofía feminista como FpN y $\mathbf{J}$ hayan sufrido ataques sobre su «filosoficidad». Durante siglos, muchas/os «otras/os» fueron consideradas/os como sujetos excéntricos al diálogo filosófico. Lo anterior se fundaba en el regateo de su plena humanidad: «varones imperfectos», «animales de cabellos largos e ideas cortas», «menores», «salvajes». ¿Cómo podían acceder al reino del pensamiento, reservado a los varones, adultos, occidentales, heterosexuales y sin discapacidades?

Las mujeres y las/os niñas/os ingresaron a la Filosofía en calidad de «polizones», y no es difícil sospechar que estuvieron «ahí» desde el principio. Sin embargo, fueron silenciadas/os de diferentes formas, quizás, incluso, se les haya «arrojado al mar» en distintas ocasiones para borrar todo rastro de su presencia. Lo que sí es innegable, es que vinieron a cuestionar las concepciones dominantes sobre filosofía, educación y ser humano. Sus voces recuerdan a la «venerable madre de todas las ciencias» algo que no debería olvidar jamás: la capacidad de pensar con autonomía, cuestionar prejuicios, argumentar con fundamentos, tomar en consideración a otros seres vivos, humanos o no, imaginar alternativas y conmoverse ante el arte y la naturaleza, es común a todas las personas, más allá de su edad, su sexo, su pertenencia étnica y su cultura. No es casualidad que la vindicación de los derechos humanos, los derechos de las mujeres, los derechos de las/os niñas/os, los derechos de los pueblos originarios, etcétera, sean una herencia del pensamiento filosófico, que ha ejercido su potencial crítico ante diversas hegemonías.

Por lo que respecta a la educación, tanto el programa de FpN y J como la teoría feminista han aportado elementos para el surgimiento de nuevas prácticas y metodologías pedagógicas. Su valoración positiva de las experiencias y los saberes de colectivos históricamente discriminados ha abierto caminos inéditos para la construcción del conocimiento. Desde las coordenadas planteadas por estas dos alternativas, el proceso educativo consiste en construir relaciones horizontales basadas en el reconocimiento recíproco, la atención al contexto, el trabajo en equipo, la empatía y la autocorrección. A. Sharp explica que las ideas y conductas sexistas se inician durante la infancia, por lo que es necesario atender dicha problemática desde los jardines de niñas/os y la educación básica. Los estereotipos de género están presentes en cada resquicio de la escuela: en las clases, en la forma en que se utiliza el lenguaje, en los libros de texto, en las ideologías y actitudes del profesorado, e, incluso, en el patio del recreo (Splitter y Sharp, 1996).

Sobre la concepción de ser humano presente en estas dos filosofías, cabe destacar que concuerdan en considerar a la persona como un ser «inacabado», cuya esencia es, precisamente, «hacerse ser». Ambas cuestionan los dualismos jerarquizados varón/mujer, adulto/ infante, mente/cuerpo, razón/emoción, teoría/práctica, conocedor/conocido, y se proponen delinear una visión integradora acerca de lo que define a las/os humanas/os, esto es, la capacidad de llegar a ser mejores de lo que son: más razonables, más empáticas/os, más creativas/os. Todo lo anterior implica, necesariamente, la visibilización de las niñas y las adolescentes y la vindicación de mejores condiciones de vida para ellas. A. Sharp dedicó la novela Hannah (2011) (y su correspondiente manual) a reflexionar sobre el concepto de persona. Si bien la perspectiva de género está presente de manera transversal en sus diferentes obras, es aquí donde aborda explícitamente el feminismo. Lo que le interesa es que las/os adolescentes exploren en comunidad temas como los estereotipos de género, la menstruación, la anorexia, el cuerpo y la sexualidad. 
La FpN y J busca la educación de personas razonables y, para lograrlo, es necesario que contribuya a crear una sociedad incluyente y plural. De acuerdo con A. Sharp, esta sociedad nueva debe reproducirse a través de mujeres y varones capaces de respetarse a sí mismas/ os y a las/os otras/os. Para ello, es imprescindible integrar el punto de vista de las mujeres al desarrollo moral de la familia y la escuela. El colectivo femenino ha estado más vinculado a la crianza y educación de las/os niñas/os, lo que, en algunos casos, ha generado el desarrollo de una mayor sensibilidad respecto a las necesidades de las/os otras/os, capacidad fundamental dentro de la ética del cuidado que reclama su universalización. Como lo expresa la autora: «llevar la filosofía a todas/os las/os niñas/os del mundo constituye una forma de ampliar las posibilidades de crear un mundo no sexista y no racista» (Sharp, 1997, 1). Filosofía feminista y FpN y J confluyen en el tipo de sociedad a la que aspiran, aunque, hasta ahora, el diálogo entre ambas, iniciado por A. Sharp, sigue siendo una asignatura sin cumplir.

Es importante destacar que, según apunta la filósofa norteamericana, la comunidad de indagación tiene el potencial para cuestionar el sexismo y promover relaciones sociales basadas en la igualdad, la inclusión, la pluralidad y la justicia. Pero, ¿qué le permite sostener esa afirmación? La idea central de A. Sharp es que la educación filosófica contribuye a formar ciudadanas/os educadas/os en los valores de la democracia. Lo anterior no puede lograrse si se deja de lado la reflexión sobre las desigualdades de género, así como los retos que plantean los derechos de las mujeres y las niñas al programa de FpN y J.

La comunidad de indagación, entendida como el proceso de hacer filosofía en el aula, ayuda a desarrollar el pensamiento complejo. Se basa en un diálogo que incluye a cada una/o de las/os participantes por igual, sin distinciones de sexo, pertenencia étnica, cultura, ni condición social; asimismo, a través de la autocorrección, promueve el crecimiento de las personas y la transformación de la sociedad; educa la razón sin dejar de lado la formación de los sentimientos; estimula la imaginación de otros mundos posibles, generando nuevos valores, ideas y alternativas para alcanzar una vida digna de ser vivida (Splitter y Sharp, 1996). Al respecto, A. Sharp argumenta lo siguiente: «Su existencia misma es un desafío al tipo de comportamiento en que se basa el sexismo, y lleva, con el tiempo, a la construcción de hábitos reflexivos basados en principios tales como la empatía, el respeto por las personas y la necesidad de pensar de manera crítica acerca de los propios supuestos, creencias y valores» $(1996,276)$.

A través de la vivencia reiterada de la indagación comunitaria, niñas/os, adolescentes y jóvenes llegan a internalizar su dignidad propia y la de las/os demás. Asimismo, adquieren experiencia de que la palabra de cada una/o forma parte del conocimiento que construyen en conjunto, y cuidan el espacio que han llegado a conformar. En este contexto, quienes han sido tradicionalmente silenciadas/os aprenden el valor y la fuerza de sus voces.

\section{4. Ética ecológica y ecofeminismo en $\mathrm{FpN}$ y J}

Las reflexiones de A. Sharp se extienden más allá de las relaciones entre personas hasta alcanzar a las plantas, los animales no humanos, los ríos, los mares, los bosques, la capa de ozono, los polos. Por un lado, el interés por la igualdad de género es una constante a lo largo de su obra; por otro, se percibe una preocupación creciente por el medio ambiente y sus habitantes. Esta pensadora se pregunta: ¿Qué lugar ocupa la ética ecológica en el proyecto de FpN y J? ¿Cuáles son los alcances de la comunidad de indagación? ¿Puede llegar a 
incluir a los no humanos? ¿Esto implicaría la redefinición de «lo humano»? ¿Cuáles son las consecuencias de la feminización de la naturaleza y de la naturalización de las mujeres? ¿De qué forma puede contribuir FpN y J a una educación ambiental con perspectiva feminista?

La reflexión ecológica se encuentra presente en el currículum de FpN y J como un tema relevante. La novela Kio y Gus (1997) y su manual correspondiente Asombrándose ante el mundo (1986) plantean consideraciones morales sobre la relación entre los animales humanos y los no humanos, así como respecto al mundo natural. A. Sharp participó en el diseño de varios de los ejercicios que se encuentran en dicho manual y, junto con J. Splitter, retomó esta problemática en La otra educación (1996). De acuerdo con nuestra filósofa, las/os niñas/ os y jóvenes de las sociedades actuales, especialmente aquellas/os que viven en contextos urbanos, perciben con dificultad un vínculo de interdependencia con la Naturaleza. Algunas/ os han nacido en ambientes donde se practica un consumo irresponsable de recursos, en tanto que muchas/os otras/os enfrentan condiciones de miseria y escasez.

Aunado a lo anterior, la crisis medioambiental es tan compleja, que resulta difícil elegir argumentos razonables entre tanta información contradictoria. Sin embargo, las/os niñas/os de hoy son quienes se convertirán en gestores, vigilantes y guardianes de la Tierra (Splitter y Sharp, 1996); por ello, es necesario que accedan a una formación ambiental sólida. El problema es que el modelo educativo basado en respuestas, muchas veces descontextualizadas y carentes de sentido para el estudiantado, está sobrepasado ante la gravedad de la situación ecológica y social. Al respecto, A. Sharp considera que la contribución más importante de FpN y J a la educación ambiental es el potencial que ofrece la comunidad de indagación para la comprensión-acción ante las graves problemáticas ambientales.

Nuestra filósofa sostiene que de las conversaciones filosóficas sobre temas ambientales podrán surgir nuevos tipos de relaciones éticas y nuevas formas de ver a la naturaleza y de relacionarnos con ella. Al pensar sobre el sentido de conceptos como cuidado, derechos de los animales no humanos y del medio ambiente, sostenibilidad, ecofeminismo, biocentrismo, antropocentrismo, entre otros, e imaginar alternativas posibles ante las problemáticas ecológicas, las aulas pueden llegar a convertirse en comunidades de indagación medioambiental. Las/os niñas/os y jóvenes participantes en los diálogos sobre filosofía y ecología tendrán la oportunidad de comprender los problemas en sus fundamentos, además de adquirir una perspectiva plural para tomar decisiones responsables (Splitter y Sharp, 1996, 300).

A juicio de A. Sharp, la perspectiva de género no puede quedar excluida de los debates actuales sobre la crisis medioambiental. Si la ética ecológica propone la extensión de los límites morales más allá de las relaciones entre seres humanos, cuestionando las dicotomías cultura/naturaleza, mente/cuerpo, razón/sentimiento, ¿cómo se justifica su olvido del dualismo varón/mujer? ¿Acaso los estereotipos de género son ajenos a dichas dualidades jerárquicas: cultura/mente/razón y naturaleza/cuerpo/sentimiento? Esta pensadora argumenta que una mirada feminista al interior de las posturas biocéntricas puede aportar elementos interesantes a la comunidad de indagación medioambiental. Más aún, según ella, el ecofeminismo ofrece algo que, por lo menos en apariencia, coincide con FpN y J: «una visión del mundo como una comunidad en la cual los individuos se definen unos a otros a través de sus relaciones recíprocas y con el suelo, el agua, la atmósfera y otros elementos no vivientes del medio ambiente que proveen el tejido que mantiene unida a la comunidad» (Mathews, citada en Splitter y Sharp, 1996, 305). 
La diferencia entre la ética ecofeminista y FpN y J reside, de acuerdo con A. Sharp, en que para las ecofeministas son más vitales las relaciones ecológicas que los individuos. Lo anterior implica una concepción sobre los alcances de la comunidad de diálogo que excede los planteamientos de FpN y J. En efecto, el proyecto educativo pensado por M. Lipman y A. Sharp sólo incluye a los seres humanos, entre quienes se pueden establecer relaciones de comunicación recíproca, lo que es difícil precisar en el caso de los otros seres vivos. A este respecto, A. Sharp se pregunta ¿hasta dónde puede abarcar la comunidad de indagación? ¿Podría incluir a los animales no humanos, a las plantas, los ríos, etcétera, además de los seres humanos? ¿Cómo impacta todo esto en el concepto de persona?

La co-fundadora de FpN no profundiza en el concepto de ecofeminismo, ni pone a discusión perspectivas diversas sobre esta corriente dentro de la teoría feminista; sin embargo, expresa su preocupación por la libertad de las mujeres ante una postura biocéntrica que coloca en el centro de sus reflexiones las relaciones ecológicas por encima de los intereses de los individuos. A. Sharp es consciente de los peligros que entraña la identificación «mujernaturaleza», así como de la vaguedad del concepto natural. Por ello, advierte a las feministas verdes: «las mujeres deben guardarse de renunciar a su individualidad cuando rechazan el individualismo y la obsesión por la autonomía que caracteriza a la tradición patriarcal» (Splitter y Sharp, 1996, 317, nota ii). Con estas palabras, la filósofa norteamericana se acerca bastante a las vertientes constructivistas del ecofeminismo, de acuerdo con las cuales, los vínculos entre el colectivo femenino y la naturaleza han sido socialmente construidos y no corresponden a ninguna esencia (Puleo, 2011).

El feminismo ecologista plantea varios retos a la educación para la sostenibilidad. Como ha apuntado la filósofa Alicia Puleo, un paso necesario es cuestionar los prejuicios androcéntricos que atraviesan la producción de conocimientos ambientales. En sus palabras: «visibilizar a las mujeres como protagonistas de alternativas ecológicas y superar el desprecio por las feminizadas actitudes relacionadas con la compasión y el cuidado son dos asignaturas pendientes de la mayor parte de los programas de educación ambiental» $(2011,23)$. Muy cercana a la propuesta educativa de FpN y J, A. Puleo subraya la importancia de la indagación en torno a conceptos como desarrollo, crecimiento, pobreza, naturaleza, medio ambiente, género, feminismo, ciudadanía, derechos sexuales y reproductivos, derechos de los animales no humanos, sin dejar de lado la formación moral de los sentimientos de compasión y la imaginación empática. Para ello, recomienda recurrir a la literatura y el cine como herramientas didácticas que potencian la imaginación de otros mundos posibles.

Por su parte, A. Sharp deja abierta la cuestión de la extensión de los límites de la comunidad de indagación al ámbito de los seres no humanos, al igual que la posibilidad de redefinir el concepto de persona a partir de las coordenadas del ecofeminismo constructivista. A mi juicio, una educación ambiental feminista podría impulsar diálogos filosóficos sobre temas como: agricultura de subsistencia, uso de la herbolaria, acceso al agua, cuidado de niñas/os, enfermas/os y adultos mayores, movimientos en defensa de la tierra y el territorio, relación entre animales humanos y no humanos, entre otros. A través de lo anterior, la comunidad de indagación incluirá a nuevos participantes, promoviendo formas alternativas de comunicación (con el huerto escolar, los animales no humanos, los ecosistemas locales) que contribuirían al replanteamiento de «lo propiamente humano». 
Es importante destacar que la educación para la sostenibilidad y más aún, desde un enfoque feminista, es una temática poco explorada dentro de la filosofía de la educación. A Sharp vislumbró la posibilidad de abordar tales cuestiones a partir de la comunidad de indagación y aportó algunas pistas teórico-metodológicas para llevarlo a cabo. Sin embargo, el desarrollo de esta línea de investigación, así como su aplicación práctica representa una tarea pendiente.

Entre las experiencias de FpN y J en este ámbito ${ }^{4}$, se encuentra el estudio realizado por Hannam y Echeverría Philosophy with teenagers (2009) ${ }^{5}$. En uno de los apartados, los autores reseñan las experiencias del Congreso Internacional para Jóvenes realizado en el año 2006, en Chiapas, México: «Paz, Justicia y sostenibilidad», donde se llevaron a cabo diálogos grupales sobre derechos de la Naturaleza y de los animales no humanos, justicia social y calidad de vida $(2009,132)$. Una de las mayores fortalezas que destacan es la diversidad de contextos de las/os jóvenes que integraron las comunidades de indagación, lo que les permitió ir construyendo respuestas provisionales y nuevas preguntas, considerando perspectivas plurales. Otro elemento clave fue que el evento se desarrolló en una región caracterizada por la presencia de culturas indígenas y una rica biodiversidad. Jóvenes de distintas etnias se integraron a las actividades, todo ello en entornos naturales, al aire libre, entre árboles y montañas ${ }^{6}$. Los testimonios de algunas/os de las/os participantes coinciden en la vivencia de una construcción comunitaria de sentido que les llevó a cuestionar el individualismo, el consumismo y la falta de empatía hacia los otros humanos y no humanos (2009, 135-136).

Para concluir este apartado y de acuerdo con la propuesta de A. Sharp sobre una educación fundada en el cuidado recíproco, el trabajo colaborativo, el diálogo incluyente y plural, la búsqueda conjunta de sentido y la revalorización de la vivencia personal como una fuente de conocimiento válida, quiero agregar algunas reflexiones en torno a mi propia experiencia en las comunidades de indagación sobre feminismo y sostenibilidad medioambiental. Como parte de un proyecto de investigación titulado «Filosofía para/ con Niñas/os y Jóvenes: aproximaciones teóricas y propuestas prácticas desde una mirada ecofeminista», que se encuentra en proceso de realización, he comenzado a implementar los diálogos filosóficos a la metodología de enseñanza/aprendizaje como parte de un curso de educación ambiental en el nivel de estudios de grado.

En la parte teórica se presentan las diferentes vertientes del ecofeminismo con el propósito de llevar a cabo una revisión crítica de las mismas, en tanto que la práctica consiste en el cuidado de un huerto de plantas medicinales. Entre las actividades didácticas se plantean temas para que las/os estudiantes investiguen antes de la sesión de diálogo: relación entre género, pobreza, acceso a los recursos naturales, producción de alimentos, conocimientos sobre herbolaria y medicina indígena. Posteriormente, les requiero que busquen en internet

4 María Teresa de la Garza Camino actualmente trabaja temas de bioética en la Universidad Nacional Autónoma de México, y ha alentado a sus estudiantes de Filosofía a producir materiales didácticos inspirados en FpN y J para abordar cuestiones de ética ecológica (entrevista realizada por Tapia González, Georgina Aimé, el 23 de junio de 2015).

5 El prólogo de este libro fue escrito por A. Sharp.

6 La filósofa norteamericana vivió durante varios años en San Cristóbal de las Casas, Chiapas, México. 
a los organismos públicos encargados de la administración y distribución del agua potable, el uso de la tierra y los recursos económicos para emprender proyectos relacionados con el campo, y redacten una relación de las personas que ocupan los puestos directivos considerando su género. Otro ejercicio que llevamos a cabo es el estudio de movimientos ambientalistas y animalistas locales desde una perspectiva de género, con el objetivo de reflexionar sobre los siguientes interrogantes: ¿Qué porcentaje de mujeres y varones integran el movimiento? ¿Qué funciones desempeñan? ¿Cómo son valoradas dichas funciones? ¿Existe alguna similitud entre lo que se observa en los movimientos ambientalistas locales y lo que sucede en otras partes del mundo? Una vez realizado lo anterior iniciamos las comunidades de indagación.

Ahora bien, conforme he ido comprendiendo que mi posición es la de un miembro más del grupo y no la de portadora de la razón, las/os estudiantes se han convertido en mis compañeras/os de viaje en una búsqueda fascinante. A través de las comunidades de indagación las/os participantes han aprendido a cuestionar los más arraigados prejuicios de género, así como los modos de vida promovidos por el capitalismo globalizado, al mismo tiempo que imaginan alternativas al respecto.

Por todo lo expuesto, considero que el proyecto filosófico de A. Sharp para la igualdad de género y la sostenibilidad medioambiental representa una aportación relevante en el laborioso camino de aprender a pensar y vivir en comunidad.

\section{Conclusiones}

En el recorrido precedente se examinaron los puntos de intersección entre FpN y J, feminismo y ética ecológica a través del estudio de la propuesta desarrollada por la filósofa norteamericana A. Sharp. Esta pensadora innovó en el currículum de FpN y J con temáticas sobre género y ecofeminismo. Sus preocupaciones principales giraron en torno a: 1) el reconocimiento de las/os niñas/os más pequeñas/os como sujetos capaces de pensamiento filosófico; 2) la visibilización de la desigualdad de género en los diferentes ámbitos escolares; 3) la educación filosófica para la igualdad entre niñas y niños; y 3) la comunidad de indagación sobre cuestiones como maltrato infantil, SIDA, diversidad étnica y cultural y crisis socioambiental.

Lo anterior me permite afirmar que los planteamientos de A. Sharp abrieron una perspectiva propia y original ante el pensamiento de M. Lipman. Por ello, continuar el diálogo entre filosofía feminista y $\mathrm{FpN}$ y $\mathrm{J}$ constituye un reto para las comunidades de indagación ante las diversas formas de sexismo presentes en la escuela desde la primera infancia. Asimismo, las ideas que apuntó sobre una educación ambiental en clave ecofeminista dejan abiertas diversas posibilidades para la redefinición de los interlocutores del diálogo filosófico en tiempos del cambio climático, sin olvidar la individualidad de las mujeres, ni la igualdad entre los sexos. De acuerdo con el ecofeminismo constructivista, esta autora comprendió los vínculos entre género y crisis socioambiental e intentó integrar estas temáticas al programa de FpN y J. A. Sharp mostró la importancia de visibilizar a las niñas y las adolescentes en un proyecto educativo que, sin ellas, no podría cumplir su objetivo, a saber: ser una guía en la búsqueda y construcción de sentido en un mundo cada vez más complejo. 


\section{Referencias}

De la Garza, Ma. T. (23 de junio de 2015), Entrevista realizada por Tapia González, Georgina Aimé, publicada en (2017). Pensar Juntos. Revista Iberoamericana de Filosofía para Niños, 1, 83-97.

Hannam, P. y Echeverría, E. (2009), Philosophy with teenagers. Nurturing a moral imagination for the 21st century. New York, Continuum.

Hoyos Valdés, D. (enero/junio, 2010), Filosofía para niños y lo que significa una educación filosófica. Discusiones filosóficas, Año 11, No 16, enero-junio, 2010. pp. 149-167.

Lipman, M. (1998), Pensamiento complejo y educación. 2a edición. Trad. de Virginia Ferrer, Madrid, Ediciones de la Torre.

Lipman, M. (1997), Kio y Gus, traducción y adaptación de Eugenio Echeverría y Claudia H. de Ponde de León, Chiapas, Centro Latinoamericano de Filosofía para Niños.

Lipman, M., Sharp, A. y Oscayan, F. (2002), La filosofía en el aula, 3a. edición, Madrid, Ediciones de la Torre.

Miranda Alonso, T. (2007), «M. Lipman: función de la filosofía en la educación de la persona razonable», en Espinosa Antón, Francisco Javier, Ocho pensadores de hoy, ed. Septem, Oviedo, pp. 173-200.

Naciones Unidas. (11 de octubre de 2014), Día Internacional de la Niña. Recuperado de: http://www.un.org/es/events/girlchild/ Fecha de consulta: 12 de agosto de 2015.

Nussbaum, M. (2010), Sin fines de lucro. Por qué la democracia necesita de las humanidades. Trad. de María Victoria Rodil, Buenos Aires/Madrid: Katz.

Pineda, D. (junio/julio, 2006), Entrevista a Matthew Lipman y a Ann Sharp. En Revista Internacional del Magisterio (21).

Recuperado de: https://cursos.aiu.edu/Filosofia\%20para\%20Ninos/PDF/Tema\%201.pdf Fecha de consulta: 2 de febrero de 21015.

Puleo, A. (2011), Ecofeminismo para otro mundo posible. Madrid, Cátedra.

Sharp, A. M. (Abril 14, 1989), Unheard Voices: Women and Children and the Evolution of Philosophy. Keynote address delivered at the Philosophy for Children, Conference, San Antonio, Texas.

Splitter, L. y Sharp, A. (1996), La otra educación. Buenos Aires, Ediciones Manantial.

Sharp, A. M. (1997), The Second Issue of Thinking devoted to the theme or Women, Feminism and Philosophy for Children. Thinking. The Journal of Philosophy for Children, 13 (1).

Sharp, A. M. (2011), Hannah, traducción y adaptación de Eugenio Echeverría, San Cristóbal de Las Casas, Chiapas, México: Centro Latinoamericano de Filosofía para Niños.

Sharp, A. M. (2014), Hospital de muñecos Y Sian y Carlota. 5a. ed, San Cristobal de la Casas, Chiapas, México, Centro Latinoamericano de Filosofía para Niños.

UNICEF. (2014), Comunicado de prensa. Casi una de cuatro adolescentes sufre violencia física. Existen alarmantes niveles de aceptación de la violencia en contra de niñas. Recuperado de: http://www.unicef.org/spanish/media/media_76221.html Fecha de consulta: 2 de septiembre de 2015.

Waksman, V. y Kohan, W. (2000), Filosofía con niños. Aportes para el trabajo en clase. Buenos Aires, Ediciones Novedades Educativas. 
